\title{
Determinación del origen de las arterias ventrales y las bifurcaciones de la aorta abdominal e iliacas en estudios de tomografía espiral multicorte
}

\author{
Juan Carlos Pari Salas ${ }^{1,2}$, Germán Augusto Vargas Olivera ${ }^{2}$, Percy Oswaldo Calderón Pérez² ,Wolfgang \\ Trillo Alvarez², Sandra Nelda Ramos Guerra², Woodward Paja Callo², María Alejandra Falconí Laos², \\ Sandra Salas Collado ${ }^{2}$ \\ ${ }^{1}$ Hospital Goyeneche. Departamento de Oncología y Radioterapia. Av. Goyeneche s/n, Arequipa, Perú. \\ 2 Universidad Católica de Santa María. Urb. San José s/n. Arequipa, Perú.
}

Recibido el 11 de diciembre del 2020. Aceptado el 15 de diciembre del 2020

DOI: https://doi.org/10.33017/RevECIPeru2020.0012/

\section{Resumen}

El estudio anatómico-morfológico de las estructuras vasculares, reviste de gran importancia, tanto en el sentido académico como para su aplicación práctica en la clínica; dichas observaciones se pueden hacer no sólo en cadáveres, sino en estudios de imágenes de sujetos vivos. El objetivo de este estudio fue determinar los orígenes de las arterias de la aorta abdominal, y las bifurcaciones de la aorta abdominal e iliaca. Se revisaron los estudios de tomografías de simulación de pacientes del servicio de Radioterapia del hospital Goyeneche, a fin de recabar información sobre el origen de las ramas ventrales de la aorta abdominal, y de la bifurcación (101 estudios). Se observó las siguientes ramas se originan de los correspondientes niveles de forma más frecuente: el tronco celíaco de T12 en , la arteria mesentérica superior de L1, las arterias renales de L1, la mesentérica inferior de L3, la bifurcación de aorta abdominal en L4, la bifurcación de la iliacas primitivas derecha e izquierda, ambas en L5. Los orígenes de la arteria mesentérica inferior y de la bifurcación de la aorta abdominal fueron los más constantes. Estos hallazgos son comparables a los que se describen en estudios previos, por lo que recomienda que se conduzcan estudios para evaluar variantes y morfología en estudios de imágenes ya hechos con algún motivo, en servicios de Radiología/ Radioterapia tanto públicos como privados.

Descriptores: Anatomía, origen, aorta abdominal, Tomografía

\section{Abstract}

The anatomical-morphological study of vascular structures holds great value, in the academic sense as in its clinical practical application; such observations can not only be made in corpses, but in image studies from living subjects. The aim of this study was to determine the origins of arteries from abdominal aorta, and its bifurcations. CT simulation studies from Hospital Goyeneche Radiotherapy Service patients were reviewed, in order to get data (101 studies). It was found that the following branches get their origins in the correspondent levels most frequently: celiac trunk in T12, superior mesenteric artery in L1, renal arteries in L1, inferior mesenteric artery in L3, abdominal aorta bifurcation in L4, and both common iliac arteries in L5. The most constant were inferior mesenteric artery origin and abdominal aorta bifurcation. These findings are similar to those already described in previous studies, therefore it is a recommendation that there should be studies made in Radiology and Radiotherapy services for evaluation of anatomical variants and morphology.

Keywords: anatomy, abdominal aorta, origin, tomography 


\section{Introducción}

Dentro del estudio anatómico-morfológico, siempre ha tenido gran interés la identificación de estructuras vasculares, sus relaciones y la ocurrencia de variaciones anatómicas; toda esta información posee gran utilidad clínica, por ejemplo, el nivel de bifurcación de la aorta abdominal revista gran importancia para la elección de los límites de los campos de irradiación pélvicos clásicos en radioterapia, y la distribución vascular del tronco celíaco posee relevancia en la programación de cirugías abdominales que involucran resección parcial o total de una o varias vísceras. Por ello, podemos encontrar descripciones a partir de series de cadáveres desde el siglo XIX, como la de Monguidi en 1893 [1], teniendo varias publicaciones más recientes. La principal dificultad en los tiempos pasados para poder estudiar dichas relaciones y variaciones anatómicas, consistía en la disponibilidad de gran número de muestras, lo cual dada la naturaleza de esta observación, podía lograrse solamente en las facultades de Medicina Humana y, con mucha más dificultad, en las morgues generales. El advenimiento de estudios como la tomografía computada o la resonancia magnética permiten evaluar el origen y relaciones de estructuras vasculares con gran precisión; además de que la evaluación es realizada una serie de imágenes, que pueden pertenecer a un sujeto vivo, aumentando así en gran medida la cantidad de muestras que se pueden conseguir. Empero, tomando en cuenta que la Tomografía computada en ese sentido, posee más idoneidad que la Resonancia Magnética, este hecho nos presenta también una barrera: no se debe irradiar a los pacientes a menos que exista una necesidad para ello, por la probabilidad de efectos estocásticos a largo plazo, riesgo que si bien es cierto ante la necesidad del estudio se supera por los beneficios que se obtienen, en ausencia de beneficio se convierte en un factor de riesgo para el paciente. Por ello, es recomendable que las imágenes que se evalúan vengan de facilidades médicas en las cuales se han realizado dichos estudios con una indicación clara; cumpliendo esta condición los centros y servicios de radiología, así como los centros y servicios de radioterapia, por la necesidad de realizar tomografías de simulación para los pacientes que reciben radioterapia conformada en $3 d$ o técnicas más avanzadas.

\section{Metodología}

Se obtuvo permiso para revisar la base de datos de tomografías de simulación del servicio de Radioterapia del Hospital Goyeneche, identificándose, desde 2016 hasta julio 2020, para buscar pacientes en los cuales se visualizara al menos uno de las arterias abdominales ventrales a estudiar. Se identificaron 101 registros $(n=101)$, para posteriormente utilizar el software de visualización de imágenes DICOM RadiantView (Medixant. RadiAnt DICOM Viewer [Software]. Version 2020.1. Mar 9, 2020. URL: https://www.radiantviewer.com).

En dicho software se utilizó el modo MPR ( MultiPlannar Reconstruction) para poder tener una vista simultánea de los tres planos, axial coronal y sagital. Seguidamente, se fueron identificando el origen de las arterias ventrales de la aorta abdominal, tomando en cuenta su relación con la vértebra o disco intervertebral correspondiente, en el plano axial. Los datos luego fueron ingresados a un software de análisis estadístico para poder realizar estadística descriptiva

\section{Resultados}

Los resultados deben ser presentados en tablas, figuras u otros que los muestren objetivamente.

Las figuras deben ser insertadas en formato jpg, las tablas usando este mismo procesador de texto. Las tablas deben ser elaboradas usando esta misma plataform MSW.

Los textos dentro de las figuras deben ser en caracteres visibles $11 \mathrm{p}$.

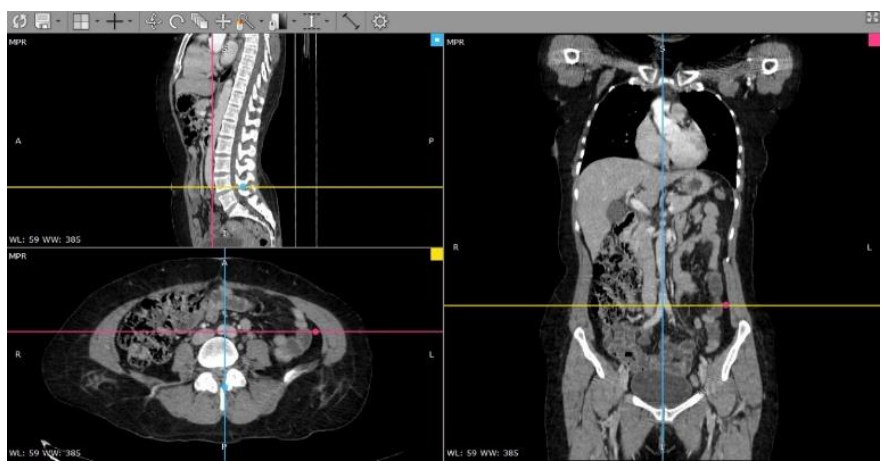

Figura 1: determinación del nivel de bifurcación de la arteria aorta abdominal en Multiplanar Reconstruction (MPR) 


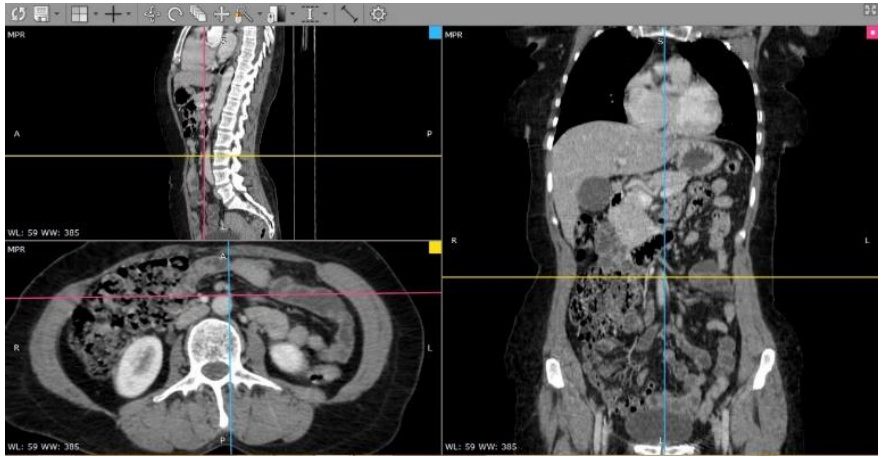

Figura 2: determinación del nivel de origen de la arteria mesentérica inferior en Multiplanar Reconstruction (MPR)

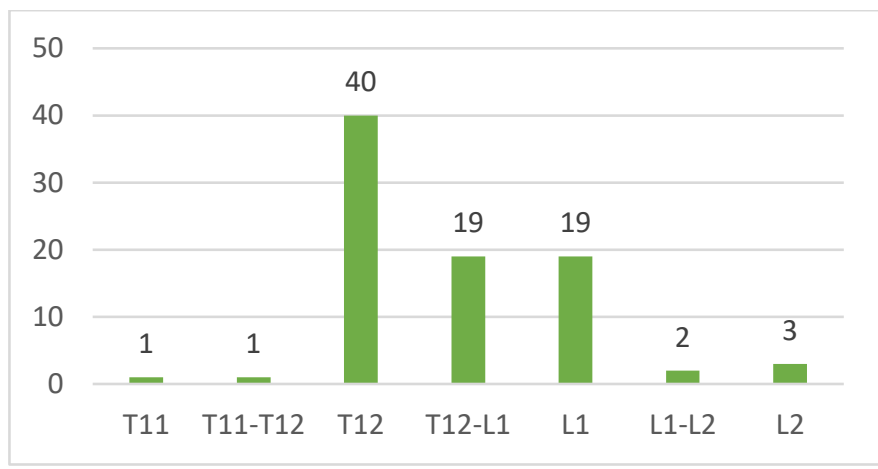

Figura 3: origen del tronco celíaco

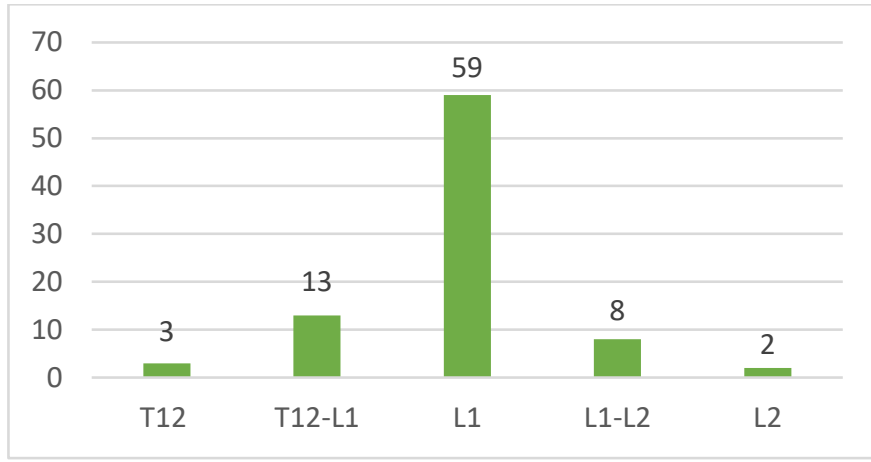

Figura 4: origen de la arteria mesentérica superior

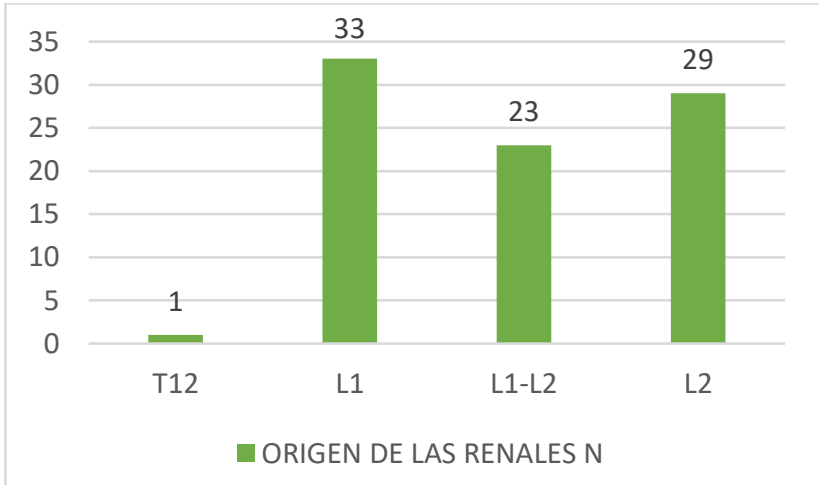

Figura 5: origen de las arterias renales

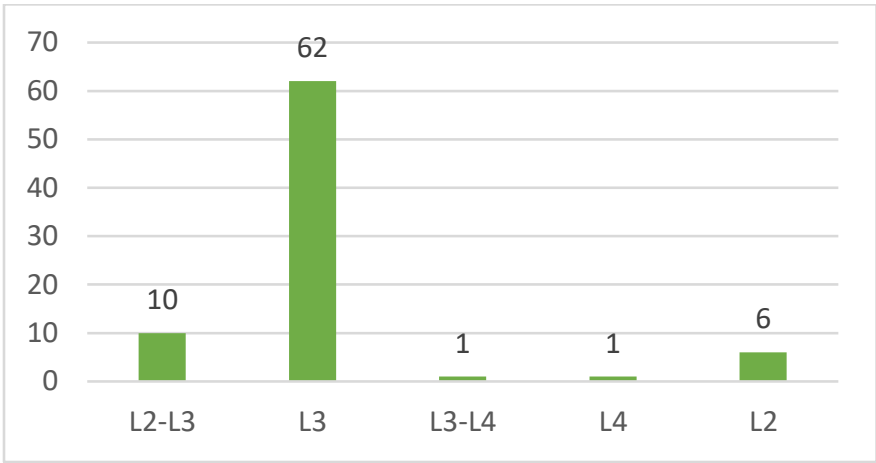

Figura 6: origen de la arteria mesentérica inferior

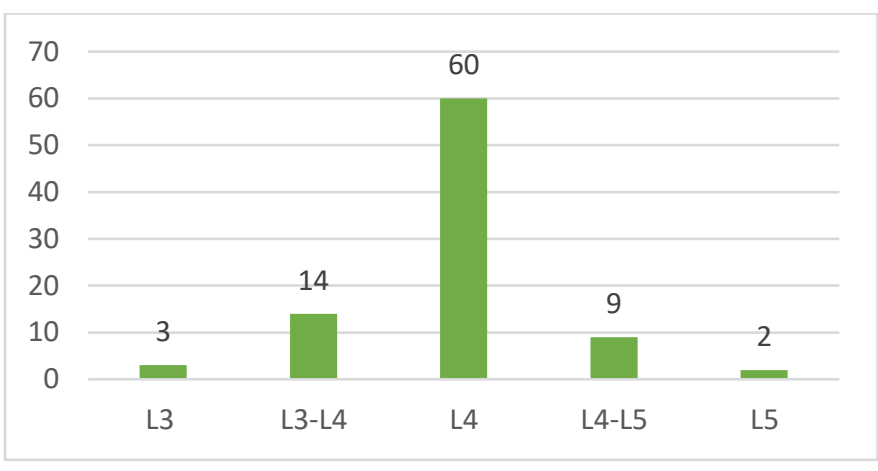

Figura 7: bifurcación de la aorta abdominal

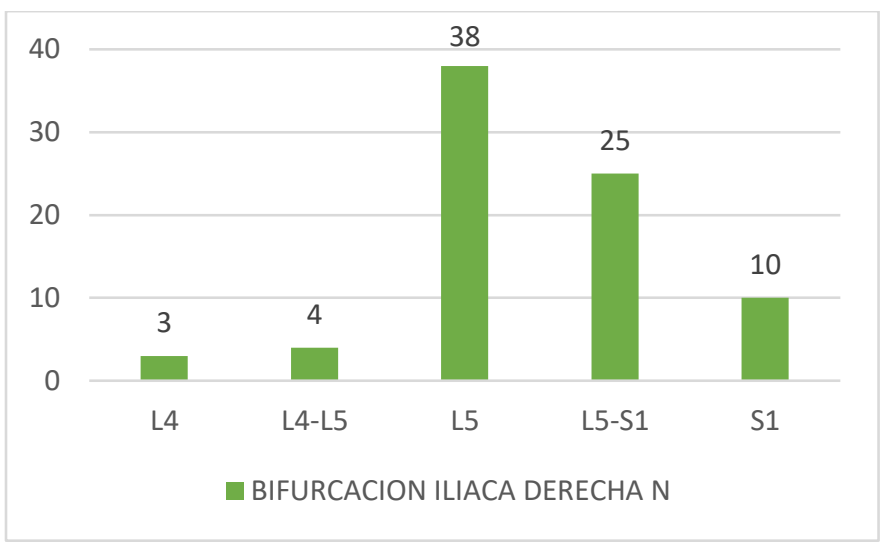

Figura 8: bifurcación de la arteria iliaca derecha

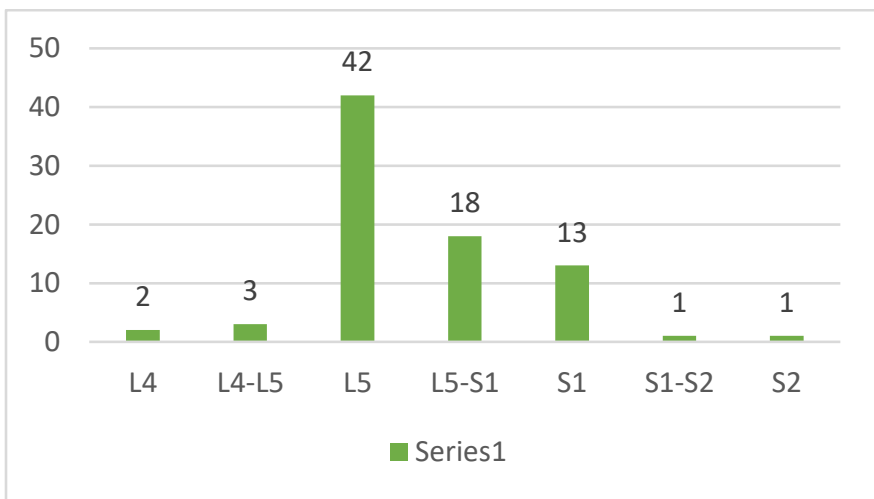

Figura 9: bifurcación de la arteria iliaca izquierda 
Tabla 1: características de la muestra

\begin{tabular}{|l|c|c|}
\hline \multicolumn{3}{|l|}{ ESTRATIFICACION POR EDAD } \\
\hline & $\mathrm{N}$ & $\%$ \\
\hline$<15$ años & 2 & 1.98 \\
\hline $16-25-$ años & 3 & 2.97 \\
\hline $26-40$ años & 14 & 13.86 \\
\hline $41-60$ años & 46 & 45.54 \\
\hline 61 años a más & 36 & 35.64 \\
\hline TOTAL & 101 & 100.00 \\
\hline ESTRATIFICACION POR SEXO \\
\hline SEXO & $\mathrm{N}$ & $\%$ \\
\hline Masculino & 32 & 32 \\
\hline Femenino & 69 & 68 \\
\hline Total & 101 & 100 \\
\hline
\end{tabular}

Tabla 2: Orígenes De Las Arterias Y Bifurcaciones

\begin{tabular}{|c|c|c|}
\hline \multicolumn{3}{|c|}{ ORIGEN DEL TRONCO CELÍACO } \\
\hline ORIGEN & $\mathbf{N}$ & $\%$ \\
\hline $\mathbf{L 2}$ & 29 & 33.73 \\
\hline T12 & 1 & 1.16 \\
\hline L1 & 33 & 38.37 \\
\hline L1-L2 & 23 & 26.74 \\
\hline TOTAL & 86 & 100 \\
\hline \multicolumn{3}{|c|}{ ORIGEN DE LA ARTERIA MESENTERICA SUPERIOR } \\
\hline ORIGEN & $\mathbf{N}$ & $\%$ \\
\hline T12 & 3 & 3.53 \\
\hline T12-L1 & 13 & 15.29 \\
\hline L1 & 59 & 69.41 \\
\hline L1-L2 & 8 & 9.42 \\
\hline L2 & 2 & 2.35 \\
\hline TOTAL & 85 & 100 \\
\hline \multicolumn{3}{|c|}{ ORIGEN DE LAS ARTERIAS RENALES } \\
\hline ORIGEN & $\mathbf{N}$ & $\%$ \\
\hline T12 & 1 & 1.16 \\
\hline L1 & 33 & 38.37 \\
\hline L1-L2 & 23 & 26.74 \\
\hline L2 & 29 & 33.73 \\
\hline TOTAL & 86 & 100 \\
\hline \multicolumn{3}{|c|}{ ORIGEN DE LA ARTERIA MESENTERICA INFERIOR } \\
\hline ORIGEN & $\mathbf{N}$ & $\%$ \\
\hline L2-L3 & 10 & 12.50 \\
\hline L3 & 62 & 77.50 \\
\hline L3-L4 & 1 & 1.25 \\
\hline
\end{tabular}

\begin{tabular}{|c|c|c|}
\hline L4 & 1 & 1.25 \\
\hline L2 & 6 & 7.50 \\
\hline TOTAL & 80 & 100 \\
\hline \multicolumn{3}{|c|}{ BIFURCACION DE LA ARTERIA AORTA } \\
\hline ORIGEN & $\mathbf{N}$ & $\%$ \\
\hline L3 & 3 & 3.41 \\
\hline L3-L4 & 14 & 15.91 \\
\hline L4 & 60 & 68.18 \\
\hline L4-L5 & 9 & 10.23 \\
\hline L5 & 2 & 2.27 \\
\hline TOTAL & 88 & 100 \\
\hline \multicolumn{3}{|c|}{ BIFURCACION ARTERIA ILIACA DERECHA } \\
\hline ORIGEN & $\mathbf{N}$ & $\%$ \\
\hline L4 & 3 & 3.75 \\
\hline L4-L5 & 4 & 5.00 \\
\hline L5 & 38 & 47.50 \\
\hline L5-S1 & 25 & 31.25 \\
\hline S1 & 10 & 12.50 \\
\hline TOTAL & 80 & 100 \\
\hline \multicolumn{3}{|c|}{ BIFURCACIÓN ARTERIA ILIACA IZQUIERDA } \\
\hline ORIGEN & $\mathbf{N}$ & $\%$ \\
\hline L4 & 2 & 2.50 \\
\hline L4-L5 & 3 & 3.75 \\
\hline L5 & 42 & 52.50 \\
\hline L5-S1 & 18 & 22.50 \\
\hline S1 & 13 & 16.25 \\
\hline S1-S2 & 1 & 1.25 \\
\hline S2 & 1 & 1.25 \\
\hline TOTAL & 80 & 100 \\
\hline
\end{tabular}

\section{Conclusiones}

La muestra presenta características peculiares, producto de su forma de obtención, siguiendo la distribución de pacientes que son sometidos a radioterapia 3D en el Departamento de Oncología, presentando características en cuanto a la distribución por edad, de acuerdo a la incidencia de neoplasias en la población.

En cuanto a la distribución por sexo, se encuentra que los estudios de pacientes de sexo femenino duplican a los de sexo masculino. Estas distribuciones hacen que no tenga utilidad evaluar la significancia estadística de las características y, por consecuencia una evaluación de los orígenes de las arterias por subgrupos.

Otros trabajos publicados $[5,6]$, coinciden en señalar que no existen diferencias en cuanto a sexo, encontrando el último de los mencionados, 
diferencias estadísticamente significativas relacionadas con la edad relativas a los orígenes de las arterias mesentérica superior y renal izquierda. Se puede observar, en las figuras correspondientes, que la mayor parte de los orígenes de arterias y bifurcaciones estudiados, parecen tener una tendencia a una distribución normal, exceptuando en este caso a las arterias renales, lo cual probablemente se deba al tamaño de la muestra; en la serie de Cauldwell y Anson [3] que constaba de 300 pacientes, se puede apreciar dicha distribución de mejor forma. El origen más frecuente del tronco celíaco se corresponde con los descrito en estudios previos, como el de Anson y Mcvay [2] o el de Cauldwell y Anson [3]; se encontraron dos casos de una arteria celiacomesentérica superior en esta serie de 101 pacientes, lo que parece estar de acuerdo con lo descrito en otros trabajos.

El origen de las arterias renales suele corresponderse con el nivel del hilio renal, siendo en este caso su identificación importante para fines de la determinación de los campos de irradiación a ganglios paraaórticos en ciertas áreas. Como con el caso de los campos pélvicos, se hace necesario el conocimiento de su origen más frecuente en el caso de la irradiación de los campos clásicos, en los cuales se suele guiar el tratamiento con imágenes de rayos $X$, estando delimitados los campos por referencias óseas. Se pude observar, en general, que el origen más constante corresponde a la arteria mesentérica inferior, lo cual es seguido por el nivel de la bifurcación de la aorta abdominal.

El origen del tronco celíaco presenta mayor variación con respecto al origen de la arteria mesentérica superior, la más cercana; el hecho de que en esta muestra haya tanta variación, y que incluso hayan dos casos con un tronco común, pone al frente el aspecto de lo relevante del conocimiento de los orígenes normales y la frecuencia de variaciones, con vistas a una aplicación práctica, como cuando se realizan cirugías oncológicas. El nivel de la bifurcación de la aorta abdominal ha sido estudiado con amplitud; Chithriki et al. [6] publican un estudio usando resonancia magnética en 441 pacientes, cuyo resultado es que en más del $60 \%$ la bifurcación se encuentra a nivel de L4, lo que se repite en los estudios antes mencionados, siendo éste más constante que los niveles de las bifurcaciones de las iliacas primitivas, que presentan una distribución con mayor variación.
Estos vasos presentan un calibre considerable y se subdividen a su vez en varias ramas, con distribución, origen y variaciones también descritas en la literatura disponible. Podemos concluir que en esta muestra de estudios, el origen de las arterias ventrales de la aorta abdominal es comparable y similar al de otras series publicadas, la mayor parte de ellas clásicas y realizadas en cadáveres. Esta correspondencia da peso a la idea de utilizar los estudios de imágenes volumétricas (como Tomografía Computada o Resonancia Magnética) para el estudio morfológico del cuerpo humano con un buen grado de certeza, lo que nos permitiría evaluar en una población mayor, la ocurrencia de variaciones anatómicas. Se recomienda que, desde los centros más interesados en estos temas, como las cátedras de Anatomía Humana de las Facultades y Escuelas de Medicina, se coordinen y conduzcan estudios para evaluar dichas variantes en estudios de imágenes ya hechos con algún motivo, en servicios de Radiología/ Radioterapia tanto públicos como privados.

\section{Referencias}

[1] MONGUIDI, Topografia del principali rami viscerali dell 'aorta abdominale. Tallardi, Milano. 1893.

[2] Barry J. Anson, Chester B. McVay. The topographical positions and the mutual relations of the visceral branches of the abdominal aorta. A study of 100 consecutive cadavers. The Anatomical Record 67(1) (1936) 7-15

[3] Earl W. Cauldwell, Barry J. Anson The visceral branches of the abdominal aorta: Topographical relationships. American Journal of Anatomy 73(1) (1943) 27-57

[4] Takahashi et al. Positional relationships among the celiac trunk, superior mesenteric artery, and renal artery observed from the intravascular space. Surg Rad Anat 35 (2013) 411-417

[5] R. Goyal, A. Aggarwal, T. Gupta, T. et al. Reappraisal of the classical abdominal anatomical landmarks using in vivo computerized tomography imaging. Surg Radiol Anat 42 (2020) 417-428.

[6] Chithriki et al. The anatomical relationship of the aortic bifurcation to the lumbar vertebrae: a MRI study. Surg Radiol Anat 24 (2002) 308-312 